\title{
Focused ion beam patterned Fe thin films: A study by selective area Stokes polarimetry and soft $x$-ray microscopy
}

\author{
P. J. Cook, T. H. Shen ${ }^{*}$ and P. J. Grundy \\ Institute of Materials Research, University of Salford, Salford, M5 4WT, England.
}

\section{Y. Im, and P. Fischer}

Center for X-ray Optics, Lawrence Berkeley National Laboratory, Berkeley CA94720, USA

\section{S. A. Morton, and A. L. D. Kilcoyne}

Lawrence Berkeley National Laboratory, 1 Cyclotron Road, Berkeley CA 94720, USA.

\begin{abstract}
We demonstrate the potential to modify the magnetic behaviour and structural properties of ferromagnetic thin films using focused ion beam "direct-write" lithography. Patterns inspired by the split-ring resonators often used as components in meta-materials were defined upon $15 \mathrm{~nm} \mathrm{Fe}$ films using a $30 \mathrm{keV} \mathrm{Ga}+$ focused ion beam at a dose of $2 \times 10^{16}$ ions $\mathrm{cm}^{-2}$. Structural, chemical and magnetic changes to the Fe were studied using transmission soft X-ray microscopy at the ALS, Berkeley CA. X-ray absorption spectra showed a 23\% reduction in the thickness of the film in the Ga irradiated areas, but no chemical change to the Fe was evident. X-ray images of the magnetic reversal process show domain wall pinning around the implanted areas, resulting in an overall increase in the coercivity of the film. Transmission electron microscopy showed significant grain growth in the implanted regions.
\end{abstract}

* Corresponding Author; E-Mail: t.shen@salford.ac.uk 
Nanoscale magnetism is currently an important area of research, with the potential to further developments in magnetic storage media and new forms of magneto-electronic devices $[1,2]$. Focused ion beam (FIB) lithography is one of many top-down techniques used to fabricate the structures required to study magnetism on the nano feature scale $[3,3 \mathrm{a}]$. FIB patterning is particularly attractive because of its "direct-write" nature. This ion irradiation patterning process from the tens of nanometers up to tens of microns features is often much faster than other multi-step lithographic techniques.

Ga ion irradiation is known to affect the magnetic properties of ferromagnetic films and in some circumstances, i.e. at high doses, render them non-magnetic [4-7]. Ozkaya et. al. show that a Ga ion dose of $1 \times 10^{16}$ ions $\mathrm{cm}^{-2}$ implanted into $30 \mathrm{~nm} \mathrm{Ni}_{81} \mathrm{Fe}_{19}$ films significantly changes the magnetic behaviour of the material, increasing its coercivity [4]. This was mostly attributed to an enlarged lattice parameter within the irradiated areas, increasing strain and impeding domain wall movement. Different sputtering rates for the Fe and Ni were seen to alter the composition of the permalloy and was also considered as a possible factor affecting the magnetic behaviour.

Conversely, Kaminsky et. al. reported that $\mathrm{Ga}$ implantation into a $15.5 \mathrm{~nm} \mathrm{Ni} 80 \mathrm{Fe}_{20}$ film with a 9 $\mathrm{nm} \mathrm{Ni}{ }_{80} \mathrm{Cr}_{20}$ capping layer reduced the coercivity, and a dose of $1 \times 10^{16}$ ions $\mathrm{cm}^{-2}$ rendered the film non-ferromagnetic at room temperature [5].

In this paper we present the effects of Ga ion irradiation on the magnetic and structural properties of Fe thin films. Irradiating a ferromagnetic film such as Fe allows us to study the effects of Ga implantation on ferromagnetism without the added complications of differential sputtering and interface mixing that can occur in more complex alloy and multi-layered films.

Several $15 \mathrm{~nm}$ Fe films were grown upon $100 \mathrm{~nm} \mathrm{Si}_{3} \mathrm{~N}_{4}$ membranes using e-beam evaporation in an ultra high vacuum system. A $30 \mathrm{keV} \mathrm{Ga}+$ FIB was then used to create $40 \times 40 \mu \mathrm{m}^{2}$ patterned areas by irradiating the films with an ion dose of $2 \times 10^{16}$ ions $\mathrm{cm}^{-2}$. A section of the pattern design is shown in Fig. 1 (c), where the dark areas represent the Ga irradiation. Surrounding 
each pattern is a $1 \mu \mathrm{m}$ wide frame, also irradiated with a dose of $2 \times 10^{16}$ ions $\mathrm{cm}^{-2}$. The patterned areas consist of arrays of 'split-ring resonators', the components that are often used to give rise to the negative magnetic permeability in meta-materials [8]. Such a pattern was chosen not only because of the potentially interesting domain structure it may produce, but also to demonstrate the versatility of the FIB and highlight its potential within the field of meta-material research. Following the patterning process, the effects of the Ga ion irradiation on the magnetic and structural properties were studied using the full field transmission soft X-ray microscope (XM-1) at beamline 6.1.2 and the scanning transmission X-ray microscope (STXM) (beamline 5.3.2) at the Advanced Light Source (ALS), Berkeley CA. respectively. X-ray magnetic circular dichroism contrast provided detailed images of the magnetic domain structure with XM-1, while the STXM was used to obtain spatially and energy dependent X-ray absorption spectra (XAS) of the patterned surface [8c]. Structural changes to the Fe were also studied using transmission electron microscopy (TEM). XM-1 is a full-field soft X-ray microscope where Fresnel zone plate optics provide a spatial resolution down to $15 \mathrm{~nm}[8 \mathrm{a}, 8 \mathrm{~b}]$. It is capable of providing magnetic and element specific information by tuning the photon energy to one of the specimen characteristic absorption edges. Using circularly polarized light emitted off-orbit from a bending magnet source to illuminate the sample through a condenser-pinhole combination one utilizes X-ray magnetic circular dichroism (XMCD), i.e. the effect that the X-ray absorption coefficient of a ferromagnetic material depends strongly upon the projection of its magnetization onto the helicity of the circularly polarized photons [10] For Fe, we use an X-ray energy of $707 \mathrm{eV}$, corresponding to the $\mathrm{L}_{3}$ absorption edge. The Fe films have an in-plane anisotropy, so to ensure that a component of the magnetization lies along the photon propagation direction, they were orientated such that the normal of the film surface made an angle of approximately 30 degrees with the incoming beam [9]. Magnetic contrast could then be observed from these oppositely orientated components. An external magnetic field was applied in-plane to saturate the film, then reversed from zero in discrete steps until saturation 
was achieved in the opposite direction. A high resolution Fresnel zone plate objective lense generates a magnified image of the film that is projected onto a a soft X-ray sensitive CCD at each field step, thus capturing a sequence of the magnetization reversal process. The process was then repeated in the opposite direction, returning the film to its original saturated state. The magnetic domain information shows up on the recorded images as additional contrast superimposed upon the structural information. By calculating the ratios between consecutive images, the structural contrast cancels out, leaving only magnetic information. It is then possible to observe the changes occurring to the domain structure between each of the field steps. This technique has been used to create the sequences in Fig. 1 (a) and (b), in which the magnetization cycle of a small section of the patterned film is shown. An outline of the pattern has been overlaid in each of the images to show the relationship between the structure of the film and the domain formation. The images (i) to (vii) in Fig. 1 (a) show the areas of the film that switch at the applied fields of $(-53,-119,-148,-170,-203,-$ 225 and -261) Oe, visible as dark regions. It is worth clarifying here that the images are not raw images, but ratios of consecutive images from the original sequence, i.e. only the changes in the magnetization structure show up.. For example, image (i) is the ratio between the original image recorded at -53 Oe and the image that precedes it, recorded at zero field. Consequently, only the changes in magnetic signal between these two field values are visible, i.e. only the areas that switch at -53 Oe. Fig. 1 (b) shows the second part of the cycle, where the images (i) to (vi) show the parts of the film switching at the applied fields of $(60,119,159,178,211$ and 243) Oe, visible as lightly shaded areas. It is immediately apparent that the Ga irradiation has not dramatically altered the ferromagnetic nature of the film, as the domains are not confined to the Fe rings but are still able to form within the irradiated areas. There is, however, a clear pinning effect created by the implanted features. This is particularly noticeable along the implanted frame that runs approximately parallel to the applied field. This is the last section of the film that is seen to reverse its magnetization in Fig. 1 (a), image (vi) and (b), image (v), at applied fields of -225 Oe and +211 Oe. It serves to 
isolate the patterned area from the surrounding Fe film, the majority of which switches during the first field step, just visible in the lower left corners of the first images in Fig. 1 (a) and (b). Within the pattern itself, the shape of the Fe rings dictates the magnetic reversal. Domains form within the lengths of Fe that lie along the applied field direction, often restricted vertically by the pinning effect of the Fe-Ga. Along the applied field direction however, the pinning is not strong enough to prevent the domains from forming between the rings. It is not clear from observing selected areas of pattern whether the Fe-Ga is magnetically harder than the as-grown Fe, since in some areas both are seen to switch at the same field. It is clear however, that the implanted regions impede the domain propagation, thus resulting in an increased coercivity when observing the patterned area as a whole.

In principle, the variations in the pixel value (proportional to the transmitted intensity) at a given location throughout the original image sequence represents a magnetic hysteresis loop, characterizing the switching behaviour of the corresponding area of the film[8b]. In order to plot the hysteresis loops, it was found necessary to correct for intensity variations across the field of view as well as from the gradually decreasing photon beam intensity, and random image shifts up to several pixels between individual frames. We therefore introduce a simple ad hoc correction method using image processing software "ImageJ" [11]. The first and last images in the sequence correspond to a magnetically fully saturated Fe film, so the ratio between these two images represents only the change in beam intensity, not part of the magnetic signal. This change is known to be approximately linear over the length of time taken to record the sequence, so we may interpolate the beam intensity for each of the intermediate images. Each image is then normalized to these calculated intensities.

Using the corrected images, a base image is created by calculating the average of the two images recorded at opposing maximum fields, where the film is fully saturated. The pixel values from small sections of Fe and Fe-Ga (indicated on Fig. 2 (a)) were measured throughout the 
sequence in order to observe the change in intensity with applied field and plotted as a fraction of the base image intensity in Fig. 2. During the sweep from 0 Oe to -884 Oe, the Fe-Ga requires one more field step than the neighbouring Fe area to reverse its magnetization This indicates restricted domain movement within this sampled area of Fe-Ga and is most likely to be a result of morphological changes and damage induced by the Ga implantation rather than chemical changes to the Fe. Inset (b) shows the XAS taken from similar areas of $\mathrm{Fe}$ and $\mathrm{Fe}-\mathrm{Ga}$, which indicates that approximately $23 \%$ of the $15 \mathrm{~nm}$ Fe film has been sputtered away in the irradiated areas. For both the Fe and Fe-Ga, the $\mathrm{L}_{2}$ and $\mathrm{L}_{3}$ absorption edges occur at $721 \mathrm{eV}$ and $708 \mathrm{eV}$ respectively. There is no apparent change in peak shape or shifting of the absorption edges within the irradiated areas, indicating that the Ga has had no noticeable effect upon chemical properties of the Fe.

TEM images comparing the structure of irradiated Fe to that of as-grown Fe are shown in Fig. 3. The grains were found to be around $25 \mathrm{~nm}$ in the Fe areas and $65 \mathrm{~nm}$ in the Fe-Ga. The difference in size is clearly visible in Fig. 3 (a), in which sections of Fe and Fe-Ga are shown. When comparing the Fe and Fe-Ga diffraction patterns in Fig. 3 (b) and (c), the difference in grain size is again evident, where the rings produced by the Fe-Ga are more discontinuous, indicative of larger grains. The diffraction rings originate from the (110), (200) and (211) planes in bcc-Fe. The lattice parameters obtained from these rings are $0.286 \mathrm{~nm}$ and $0.283 \mathrm{~nm}$ for the Fe and Fe-Ga respectively. This small decrease in lattice parameter is not significantly greater than the experimental error that could be expected in recording the diffraction patterns.

The increase in grain size within the implanted region is a result of the hundreds of displaced Fe atoms per incident Ga ion, creating an ion induced annealing effect. Using the "SRIM-2008" software, it is possible to simulate the final ion distribution and kinetic phenomena involved during the ion implantation process [12]. The simulation estimates around $700 \mathrm{Fe}$ displacements per Ga ion, so with the high ion dose used here, each Fe atom may have been displaced many times over. The final ion distribution shows that approximately $60 \%$ of the Ga ions 
stop within the $\mathrm{Fe}$, with the peak concentration at a depth of around $10 \mathrm{~nm}$. On average, Ga is estimated to constitute approximately $12 \%$ to the film in the irradiated areas.

To summarize, Ga ion implantation can significantly alter the magnetic properties of Fe without complete removal of the film. The domain formation during the magnetization reversal in the patterned film is determined by the magnetostatic energy of the Fe rings, where it is energetically favourable for the domains to form within the longer sections parallel to the applied field. This effect would be more readily seen in a simpler pattern such as a grid or an array of dots; we will be addressing this further in future publications. The presence of the Fe-Ga hinders the domain propagation by providing pinning sites for the domain walls leading to an increase in coercivity within the patterned area. The pinning effect may be caused by the structural changes to the implanted areas, where significant enlargement of the Fe grains occurs.

\section{Acknowledgements}

The authors wish to acknowledge the EPSRC for their support. This work was supported by the Director, Office of Science, Office of Basic Energy Sciences, Materials Sciences and Engineering Division, of the U.S. Department of Energy under Contract No. DE-AC02-05-CH11231. We would like to thank the staff at the ALS and CXRO, Berkeley, for their kind help and assistance during the experiments. We also thank the Pro-Vice-Chancellor Research of Salford University for providing the special travel grant. 


\section{References}

1. Z. Z. Bandic, E. A. Dobisz, T. W. Wu and T. R. Albrecht, Solid State Technology 49 (9), S7 (2006).

2. C. A. F. Vaz, T. J. Hayward, J. Llandro, F. Schackert, D. Morecroft, J. A. C. Bland, M. Kläui, M. Laufenberg, D. Backes, U. Rüdiger, F. J. Castano, C. A. Ross, L. J. Heyderman, F. Nolting, A. Locatelli, G. Faini, S. Cherifi and W. Wernsdorfer, Journal of Physics-Condensed Matter 19 (25), - (2007).

3. J. Fassbender, J. McCord, Journal of Magnetism and Magnetic Materials 320 (3-4), 579-596 (2008).

3a G.J. Kusinski, K.M. Krishnan, G. Denbeaux, G. Thomas, B.D. Terris, and D. Weller "Magnetic imaging of ion-irradiation patterned Co/Pt multilayers using complementary electron and photon probes" Applied Physics Letters, 79(14), 2211 (2001)

4. D. Ozkaya, R. M. Langford, W. L. Chan and A. K. Petford-Long, Journal of Applied Physics 91 (12), 9937-9942 (2002).

5. W. M. Kaminsky, G. A. C. Jones, N. K. Patel, W. E. Booij, M. G. Blamire, S. M. Gardiner, Y. B. Xu and J. A. C. Bland, Applied Physics Letters 78 (11), 1589-1591 (2001).

6. N. Owen, H. Y. Yuen and A. Petford-Long, Ieee Transactions on Magnetics 38 (5), 25532555 (2002).

7. R. Hyndman, P. Warin, J. Gierak, J. N. Chapman, J. Ferré, J. P. Jamet, V. Mathet, C. Chappert, Journal of Magnetism and Magnetic Materials 240 (1-3), 50-52 (2002).

8. C. M. Soukoulis, S. Linden, M. Wegener, Science 315, 47-49 (2007).

8a W. Chao, B.H. Harteneck, J.A. Liddle, E.H. Anderson and D.T. Attwood, "Soft x-ray microscopy at a spatial resolution better than $15 \mathrm{~nm}$ " Nature, 435, 1210 (2005)

8b D.-H. Kim, P. Fischer, W. Chao, E. Anderson, M.-Y. Im, S.-C. Shin, and S.-B. Choe "Magnetic soft Xray microscopy at $15 \mathrm{~nm}$ resolution probing nanoscale local magnetic hysteresis (invited)" J. Appl. Phys., 99, 08H303 (2006) 
8c. A.L.D. Kilcoyne, T. Tyliszczak, W. Steele, S. Fakra, P. Hitchcock, K. Franck, E.H. Anderson, B. Harteneck, E.G. Rightor, G.E. Mitchell, A.P. Hitchcock, L. Yang, T. Warwick and H. Ade, "Interferometer Controlled Scanning Transmission X-ray Microscopes at the Advanced Light Source”, J. Synchrotron Radiat. 10(2): 125-136 (2003)

9. P. Fischer, T. Eimuller, G. Schutz, M. Kohler, G. Bayreuther, G. Denbeaux and D. Attwood, Journal of Applied Physics 89 (11), 7159-7161 (2001).

10. P. Fischer, D.H. Kim, W. Chao, J.A. Liddle, E.H. Anderson, D.T. Attwood "Soft X-ray microscopy of nanomagnetism" Materials Today, 9(1-2), 26 (2006)

11. W. S. Rasband, ImageJ, U. S. National Institutes of Health, Bethesda, Maryland, USA, http://rsb.info.nih.gov/ij/, 1997-2008.

12. J. F. Ziegler, SRIM-2008, http://www.srim.org 


\section{Captions}

Figure 1. Magnetization reversal cycle of a section of patterned Fe, with the applied field in the horizontal direction. The images show the change in domain structure between successive field steps, with an overlay of the pattern design as a guide. An in-plane field of +913 Oe was first applied to saturate the film, then swept from zero field to 884 Oe (a), and then zero field to +913 Oe (b). The pattern design is shown in (c), with the dark areas representing the Ga implantation.

Figure 2. Partial magnetic hysteresis loops from the two sections of the film highlighted in (a). The dashed lines are present only as guides for the eye and do not represent actual data. The X-ray absorption spectra for Fe and Fe-Ga are shown in (b?).

Figure 3. (a) TEM image showing the effect of Ga irradiation on the grain size of the Fe. The grains in the as-grown and irradiated Fe measure approximately $25 \mathrm{~nm}$ and $65 \mathrm{~nm}$ respectively. Selected area diffraction patterns from the Fe (b) and Fe-Ga (c) show the (110), (200) and (211) rings. 

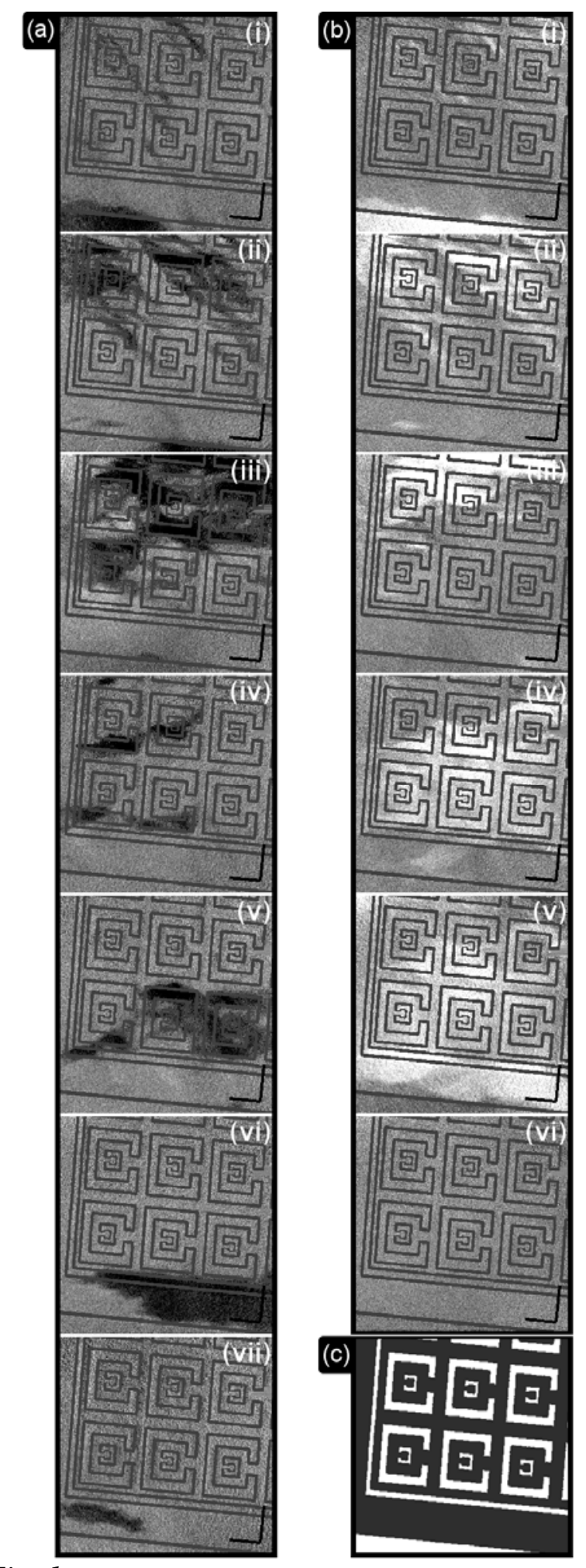

Fig. 1 


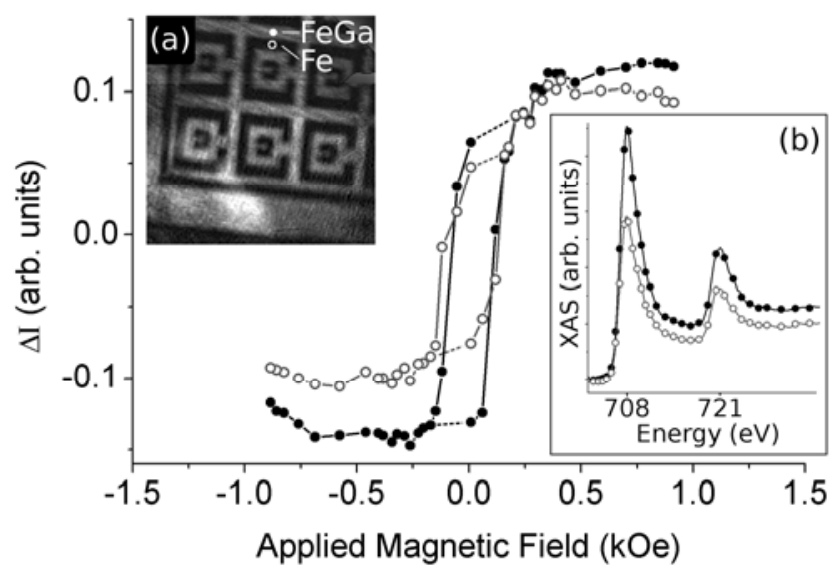

Fig. 2 


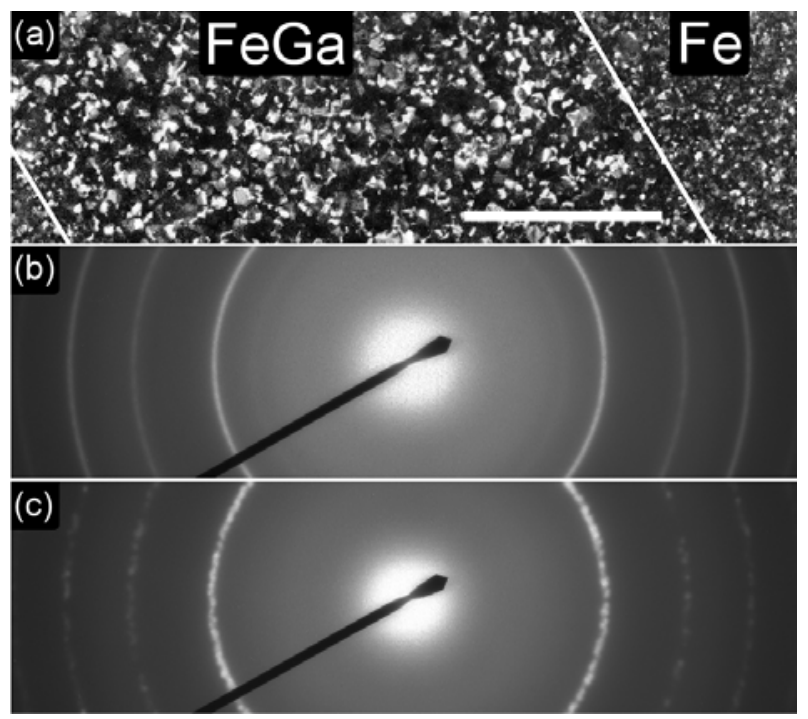

Fig. 3 


\section{SCLAI MER}

This document was prepared as an account of work sponsored by the United States Government. While this document is believed to contain correct information, neither the United States Government nor any agency thereof, nor The Regents of the University of California, nor any of their employees, makes any warranty, express or implied, or assumes any legal responsibility for the accuracy, completeness, or usefulness of any information, apparatus, product, or process disclosed, or represents that its use would not infringe privately owned rights. Reference herein to any specific commercial product, process, or service by its trade name, trademark, manufacturer, or otherwise, does not necessarily constitute or imply its endorsement, recommendation, or favoring by the United States Government or any agency thereof, or The Regents of the University of California. The views and opinions of authors expressed herein do not necessarily state or reflect those of the United States Government or any agency thereof or The Regents of the University of California. 